\title{
PRODUTIVIDADE DE CANA-DE-AÇÚCAR COM A UTILIZAÇÃO DE DOSES E FONTES DE GESSO EM UM LATOSSOLO NO ESTADO DE PERNAMBUCO
}

\author{
LIMA, Fábio Henrique Soriano ${ }^{1}$ \\ PEREIRA JUNIOR, Caetano Cláudio ${ }^{2}$ \\ MACIEL JUNIOR, Vinícius Antônio ${ }^{2}$ \\ PEREIRA, Sônia Valéria ${ }^{3}$
}

Recebido em: 2009.08 .16

Aprovado em: 2010.23.03

ISSUE DOI: $10.3738 / 1982.2278-270$

RESUMO: Neste trabalho são avaliadas as respostas, em produtividade, do primeiro corte (cana planta) da cultura da cana-de-açúcar, utilizando a variedade SP79-1011 em um Latossolo vermelho-amarelo, mediante a aplicação de doses de gesso. Estabelecendo um estudo que utiliza duas fontes de gesso aqui chamadas de Gesso Mineral (GM) e Gesso Reciclado (GR). O estudo foi conduzido em área experimental implantada no campo agrícola da Usina Cucau, no município de Rio Formoso-PE. Além do peso após o corte de cada parcela com seu respectivo tratamento, foi avaliado o percentual de fibra, a quantidade de ATR (Açucares Totais Recuperáveis) e a TPH (Tonelada de Pol por hectare), mediante análises tecnológicas da cana produzida, permitindo assim uma avaliação quantitativa e qualitativa da matéria prima (cana-de-açúcar). Com relação ao peso por parcela a testemunha (zero de gesso) apresentou maior valor seguido pela dose 2 de ambos tratamentos (GM e GR). A maior dose de GR resultou no maior teor de fibra e as doses 1 e 2 do GM junto com a testemunha demonstraram os maiores teores subsequentes. As doses 1 do GM e 4 do GR tiveram as melhores respostas em ATR e a dose 2 do GA e 4 do GR em Tonelada de Pol/ ha.

Palavras-chave: Cana-de-açúcar, Produtividade, Gesso.

SUMMARY: This work is about the answers of yield on the first cut of the sugar cane (plant cane), using the varieties SP79-1011 on an Oxisol red-yellow (Latossolo vermelho-amarelo in Portuguese), by the application of gypsum doses. Establishing a study that uses two gypsum sources, here called Mineral Gypsum (MG/ GM in Portuguese) and Recycled Gypsum (RG/GR in Portuguese). The study was lead in a experimental area, implanted on the Usina Cucau's agricultural field, in Rio Formoso City - PE. Beside the weight after the cut of each plot with the respective treatment, was valued the percentage of fiber the amount of TSR - Total Sugars Recovered (ATR in Portuguese) and Tons of Pol per Hectare (TPH), by technological analysis of the cane produced, permitting a quantitative and qualitative valuation of raw material (sugar cane). In relation of (about) the weight per plot, the witness (zero gypsum) presented a major value followed by the dose 2 for both treatments (GM and GR). The major dose of GR resulted on a major tenor of fiber, and the doses 1 and 2 of GM together with the witness showed the major tenors sub-sequent. The doses 1 of GM and dose 4 of GR had the best answers about ATR and the dose 2 of GM and dose 4 of GR best answers about TPH.

Keywords: Sugar Cane, Productivity, Gypsum.

\section{INTRODUÇÃO}

A Cana-de-açúcar tem sua produção destinada, principalmente, à indústria do açúcar e do álcool.

\footnotetext{
${ }^{1}$ Pós-graduandos do Curso de Pós-graduação Lato sensu de Gestão e Tecnologia Sucroalcooleira, oferecido pela Faculdade Dr. Francisco Maeda-FAFRAM/FE. Ituverava/SP.

${ }^{2}$ Orientador e Professor da Faculdade Dr. Francisco Maeda - FAFRAM/FE, Ituverava-SP.

${ }^{3}$ Dr $^{\mathrm{a}}$. em Botânica - Instituto de Tecnologia de Pernambuco - ITEP. Recife, PE.
} 
Dentre os mais diversos produtos agrícolas destinados à industrialização, destaca-se pela tradição, pela relevância sócio-econômica, pela sua função energética e pela distribuição geográfica em toda a Zona da Mata do Estado de Pernambuco, compondo aproximadamente $40 \%$ da economia do estado.

Pode-se dizer, ainda, que no Brasil a cana-de-açúcar deu sustentação ao seu processo de colonização, tendo sido a razão de sua prosperidade nos dois primeiros séculos.

Foi na Capitania de Pernambuco, pertencente a Duarte Coelho, onde se implantou e floresceu o primeiro centro açucareiro do Brasil, motivado por três aspectos importantes: a habilidade e eficiência do donatário; a terra e clima favorável à cultura da cana; e a situação geográfica de localização mais próxima da Europa em relação à região de São Vicente (São Paulo), outro centro que se destacou como iniciador de produção de açúcar do Brasil Colonial.

Porém um dos entraves a um possível acréscimo na produção sucroalcooleira da região da Zona da Mata Sul do estado de Pernambuco é a baixa capacidade de exploração de água e nutrientes do solo, ocasionada pelo pequeno desenvolvimento sistema radicular da planta.

Esta pode ser explicada, basicamente, pela fitotoxidade ocasionada pela forte presença do elemento $\mathrm{Al}^{3+}$, que aumenta a acidez trocável do solo formando uma barreira química que impede um maior desenvolvimento radicular da cultura.

Segundo Landell et al. (2003), a condição química do horizonte subsuperficial é determinante da produtividade de cana-de-açúcar, ampliando-se essa correlação com a produtividade $(\mathrm{TCH})$ com o avançar dos cortes.

Caires et al (1999), em revisão sobre o assunto, relatam que as limitações causadas pela acidez do subsolo à produtividade agrícola têm sido amplamente divulgadas na literatura e esses resultados mostram que as limitações se dão, sobretudo no crescimento do sistema radicular das culturas com efeitos diretos na absorção de água e nutrientes.

Portanto, ações de pesquisa que possam atuar na melhoria do ambiente radicular da cana-de-açúcar em solos da região, certamente irão proporcionar um significativo aumento de produtividade.

Um dos resíduos mais utilizados como corretivo de solos salinos sódicos e subsolos ácidos, é o gesso. A aplicação do gesso promove a lixiviação de cálcio, tendo como consequência o enriquecimento da camada subsuperficial de solo e a redução da saturação do $\mathrm{Al}^{3+}$ tóxico (QUAGGIO, 2000).

Aliado a fitotoxidade dos solos em questão, também se tem como questão motivadora a este estudo a resolução 307/02 do CONAMA, que responsabiliza aos seus geradores, a 
destinação de resíduos da construção civil, entre eles o gesso. Sendo esta questão do despejo indevido, um problema ambiental.

Nesta situação, e baseado em outros estudos que consideram o gesso fonte de cálcio e enxofre, elementos essenciais para o crescimento das plantas, cuja carência é muito frequente em solos brasileiros.

Visando indicar tecnologias que permitam o melhor aproveitamento de recursos aliado ao aumento de produtividade, além da preocupação com a conservação e preservação do meio ambiente.

Foi estabelecido o estudo utilizando o gesso residual ou reciclado (GR) oriundo da construção civil e o gesso minerado (GM), originário da reação de ácido sulfúrico sobre a rocha fosfatada, para produzir ácido fosfórico, que segundo Raij (1988), é citado como possível corretivo agrícola e insumo utilizado no cultivo da cana de açúcar.

Este estudo tem como objetivo demonstrar a possível utilização do gesso como corretivo de subsolos ácidos e as respostas proporcionadas por este uso à produtividade da cultura da cana-de-açúcar, além de indicar uma fonte alternativa de gesso (GR) que possa também ser utilizada assim como a tradicionalmente utilizada (GM).

\section{MATERIAL E MÉTODOS}

\section{Coleta e beneficiamento das fontes de gesso}

Na coleta, beneficiamento (Figuras 1, 2 e 3) e caracterização das fontes de gesso, (Tabelas 1 e 2), foram executadas as determinações de Umidade, Sulfato e Cálcio de acordo com ABNT-NBR 12130, 1991 e análise granulométrica segundo metodologia ABNT-NBR 6947, 2001. Tais análises foram efetuadas pelo Instituto de Tecnologia de Pernambuco (ITEP) no: Núcleo Tecnológico do Gesso - NTG e nos Laboratórios especializados em Ensaios e Analises Inorgânicas - LEAI; de Química Ambiental - LQA e Biotecnologia Ambiental LABTAM.

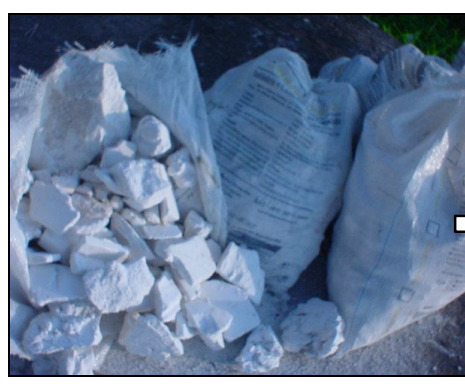

Figura 1. Resíduo de Gesso.

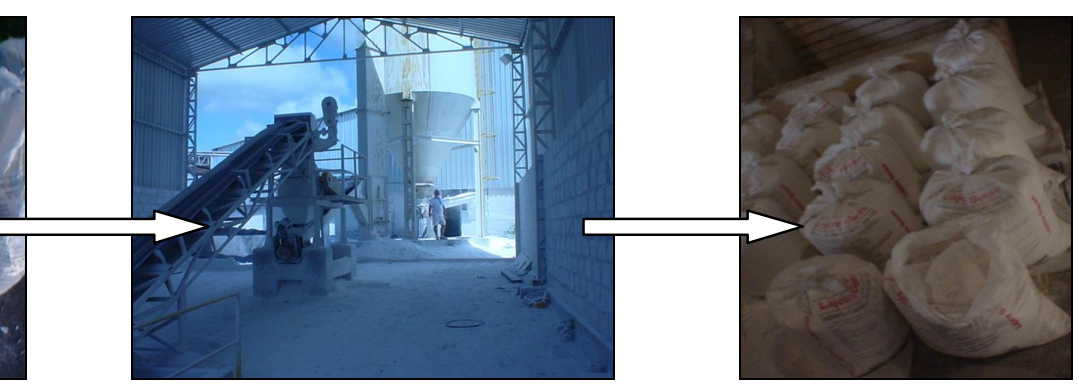

Figura 2. Beneficiamento do resíduo de gesso de construção.
Figura 3. Gesso Reciclado. 


\section{Parâmetros Analisados das Fontes de Gesso}

Tabela 1. Teores de umidade em base gravimétrica, de sulfato e de cálcio das amostras de gesso reciclado e minerado.

\begin{tabular}{cccc}
\hline D E T E R M I N A Ç Õ E S & GR & GM \\
\cline { 2 - 4 } & & 00,16 & 0,77 \\
Umidade $\left(\mathbf{a ~ 4 5 0}^{\circ} \mathbf{C}\right)$ & 41,70 & & 37,04 \\
Sulfato $\left(\mathbf{e m ~ S O}_{\mathbf{3}}\right)$ & 22,86 & & 20,34 \\
Cálcio $(\mathbf{e m ~ C a ) ~}$ & &
\end{tabular}

GR - Gesso Reciclado; GM - Gesso Mineral.

Tabela 2. Caracterização granulométrica via seca das amostras de Gesso Reciclado e Mineral. (NBR 6946/87)

\begin{tabular}{|c|c|c|c|c|c|c|c|}
\hline \multirow{2}{*}{$\begin{array}{c}\text { Peneira } \\
\text { ABNT } \\
\mathbf{N}^{\mathbf{0}} \\
\end{array}$} & \multirow{2}{*}{$\begin{array}{c}\text { Abertura } \\
\text { (mm) }\end{array}$} & \multicolumn{2}{|c|}{ Material retido } & \multicolumn{2}{|c|}{ Mat. acum. retido } & \multicolumn{2}{|c|}{$\begin{array}{c}\text { Mat. acum. } \\
\text { peneirado }\end{array}$} \\
\hline & & GR & GM & GR & GM & GR & GM \\
\hline 10 & 2,00 & 0,22 & 0,47 & 0,22 & 0,47 & 99,78 & 99,53 \\
\hline 20 & 0,84 & 1,80 & 9,39 & 2,02 & 9,86 & 97,98 & 90,14 \\
\hline 50 & 0,30 & 8,77 & 28,93 & 10,79 & 38,79 & 89,21 & 61,21 \\
\hline
\end{tabular}

GR - Gesso Reciclado; GM - Gesso Mineral.

\section{Caracterização da Área}

Inicialmente à implantação do experimento foi feita a caracterização do solo da área experimental (Tabela 3).

Tabela 3. Caracterizarão física e química do solo coletado na área experimental nas profundidades de 0-20, 20-40, 40-60, 60-80 cm.

\begin{tabular}{|c|c|c|c|c|c|c|c|c|c|c|c|}
\hline Prof. & $\begin{array}{c}\text { pH } \\
(\mathbf{H 2 O})\end{array}$ & $\mathbf{P}$ & Ca & $\mathrm{Ca}+\mathrm{Mg}$ & Al & $\mathbf{K}$ & $\mathbf{N a}$ & CTC & S.B. & $\mathbf{m}$ & Argila \\
\hline $\mathrm{Cm}$ & & $\mathrm{mg} / \mathrm{dm}^{3}$ & & & $-\mathrm{cr}$ & olc dn & & & & & \\
\hline $0-20$ & 5,28 & 27,77 & 4,08 & 5,98 & 0,28 & 0,14 & 0,08 & 6,47 & 6,20 & 3,97 & 21,24 \\
\hline $20-40$ & 4,45 & 0,30 & 0,70 & 1,64 & 1,05 & 0,10 & 0,06 & 2,85 & 1,80 & 37,91 & 26,73 \\
\hline $40-60$ & 4,30 & 0,10 & 0,20 & 0,90 & 1,48 & 0,10 & 0,04 & 2,52 & 1,04 & 59,64 & 24,85 \\
\hline $60-80$ & 4,25 & 0,08 & 0,23 & 0,65 & 1,40 & 0,24 & 0,04 & 2,33 & 0,93 & 60,34 & 27,93 \\
\hline
\end{tabular}

\section{Delineamento Experimental}

O experimento foi instalado no campo da usina Cucau com cerca de 0,26 hectare formado por 3 blocos com 11 tratamentos sendo 5 doses, duas fontes do gesso e uma testemunha (com apenas calagem e adubações) que resultou em um esquema fatorial do tipo $[(5 \times 2)+1] \times 3$, disposto em um delineamento experimental em blocos casualizados, mediante sorteio aleatório com relação a localização das parcelas e seus tratamentos na área.

As parcelas experimentais foram formadas por 6 sulcos de plantio de 10 metros lineares e 1,0 metro de espaçamento entre sulcos contendo $50 \mathrm{~m} 2$ de área útil, distanciadas com 2 metros radiais uma das outras. 
Foram testadas duas fontes de gesso em cinco níveis diferentes, baseados na necessidade de calagem $(0,0 ; 0,25 ; 0,50 ; 0,75 ; 1,00$ e 1,50 x a NC), correspondendo, respectivamente as doses: $1=0.0 ; 2=0,74 ; 3=1,48 ; 4=2,21 ; 5=2,950 ; 6=4,45 \mathrm{t} /$ ha de gesso (tabela 4).

Tabela 4. Níveis e doses de gesso e necessidade de calcário utilizado nas parcelas do experimento.

\begin{tabular}{|c|c|c|}
\hline Níveis de gesso (\% de NC) & Doses (t/ha) & Doses (kg/parcela) \\
\hline $1=00,00$ & 0,00 & 0,00 \\
\hline $2=25,00$ & 0,74 & 3,70 \\
\hline $3=50,00$ & 1,48 & 7,40 \\
\hline $4=75,00$ & 2,21 & 11,05 \\
\hline $5=100,0$ & 2,95 & 14,75 \\
\hline $6=150,0$ & 4,45 & 22,25 \\
\hline Necessidade de calagem (ton/ha) & & \\
\hline Quantidade de calcário (ton/ha) & & \\
\hline
\end{tabular}

Obs.: parcelas com $50 \mathrm{~m} 2$.

\section{Implantação do Experimento}

As parcelas experimentais situadas no lote "EL 58" do Engenho El Dourado da usina Cucau possuem como características edáficas um Latossolo Vermelho-amarelo com topografia razoavelmente plana, em condição de chã.

Em todas as parcelas o solo foi corrigido com calcário, conforme a necessidade de calagem (NC) da camada 0-20 cm de profundidade, utilizando o método da neutralização do alumínio trocável ou elevação dos teores trocáveis de cálcio e magnésio. Em seguida foram realizadas as adubações de fundação e cobertura das parcelas, conforme o Boletim de Recomendação de Corretivos e Fertilizantes para o Estado de Pernambuco (CAVALCANTI et al., 1998).

\section{Corte (colheita), Pesagem e Avaliação da Cana-de-açúcar.}

O corte, ou colheita, da área experimental foi executado manualmente com a cana queimada, 16 meses após o plantio da cana.

A pesagem foi feita com o auxílio de um aparelho conhecido como dinamômetro, pesando os feixes de cana cortados de cada parcela obtendo como resultado o peso em $\mathrm{Kg}$ de cana em cada parcela com seus respectivos tratamentos.

As análises tecnológicas foram efetuadas no laboratório da unidade industrial da Usina Cucau. Nelas foram determinados o Peso do Bulbo Úmido (PBU), a Leitura Sacarimétrica e o Brix do Caldo. A partir desses foram calculados o Percentual de Fibra, o teor de Açúcares Totais Recuperáveis (ATR) em kg por tonelada de cana e a Tonelada de Pol por hectare (TPH). 


\section{RESULTADOS E DISCUSSÃO}

Os valores resultantes das avaliações foram devidamente agrupados e analisados estatisticamente utilizando programa de computação SAEG ${ }^{\circledR}$.

Foram realizadas análises de comparação de médias e teste de Tukey a $5 \%$ de probabilidade. Os dados foram dispostos em forma de tabela apresentada a seguir.

Tabela 5. Comparativo de médias segundo teste de Tukey a 5\% de probabilidade e seus respectivos coeficientes de variação (C.V.).

\begin{tabular}{|c|c|c|c|c|c|c|c|c|}
\hline \multirow[t]{2}{*}{ Doses } & \multicolumn{2}{|c|}{ Peso parcela $(\mathrm{Kg})$} & \multicolumn{2}{|c|}{ Fibra (\%) } & \multicolumn{2}{|c|}{$\operatorname{ATR}(\mathrm{Kg} / \mathrm{Ton})$} & \multicolumn{2}{|c|}{ TPH } \\
\hline & GR & GM & GR & GM & GR & GM & GR & GM \\
\hline 0 & \multicolumn{2}{|c|}{$815,00 \mathrm{a}$} & \multicolumn{2}{|c|}{$12,56 \mathrm{a}$} & \multicolumn{2}{|c|}{$145,02 \mathrm{a}$} & \multicolumn{2}{|c|}{$16,97 \mathrm{a}$} \\
\hline 1 & $761,67 \mathrm{a}$ & $756,67 \mathrm{a}$ & 12,19 & $12,58 \mathrm{a}$ & $143,26 \mathrm{a}$ & $155,71 \mathrm{a}$ & $15,50 \mathrm{a}$ & $16,94 \mathrm{a}$ \\
\hline 2 & $795,00 \mathrm{a}$ & $803,33 \mathrm{a}$ & 12,42 & $12,63 \mathrm{a}$ & $135,24 \mathrm{a}$ & $152,06 \mathrm{a}$ & $14,79 \mathrm{a}$ & $17,62 \mathrm{a}$ \\
\hline 3 & 743,33 a & $746,67 \mathrm{a}$ & 12,09 & $12,03 \mathrm{a}$ & $138,90 \mathrm{a}$ & $151,19 \mathrm{a}$ & $14,67 \mathrm{a}$ & $16,29 \mathrm{a}$ \\
\hline 4 & $778,33 \mathrm{a}$ & $766,67 \mathrm{a}$ & 12,54 & $12,28 \mathrm{a}$ & $153,51 \mathrm{a}$ & $142,88 \mathrm{a}$ & $17,21 \mathrm{a}$ & $15,70 \mathrm{a}$ \\
\hline 5 & 733,33 a & $775,00 \mathrm{a}$ & 12,63 & $12,36 \mathrm{a}$ & 146,76 a & $152,23 \mathrm{a}$ & $15,51 \mathrm{a}$ & $17,02 \mathrm{a}$ \\
\hline C. V. & \multicolumn{2}{|c|}{$5.702 \mathrm{a}$} & \multicolumn{2}{|c|}{$2.999 \mathrm{a}$} & \multicolumn{2}{|c|}{$9.201 \mathrm{a}$} & \multicolumn{2}{|c|}{$11.444 \mathrm{a}$} \\
\hline
\end{tabular}

Valores seguidos de mesma letra na linha não diferem estatisticamente entre si, mediante o teste de Tukey a $5 \%$ de probabilidade.

Nas figuras 4 e 5 são apresentados, em forma de gráficos, respectivamente os valores de $\mathrm{kg}$ de cana-de-açúcar e os teores de fibra produzidos em cada parcela experimental, referente a cada um dos tratamentos utilizados.

Com o auxílio dos gráficos é possível observar que há uma correlação entre os acréscimos em peso $(\mathrm{kg})$ e os teores de fibra (\%) na cana produzida, referente a cada uma das doses de gesso utilizadas.

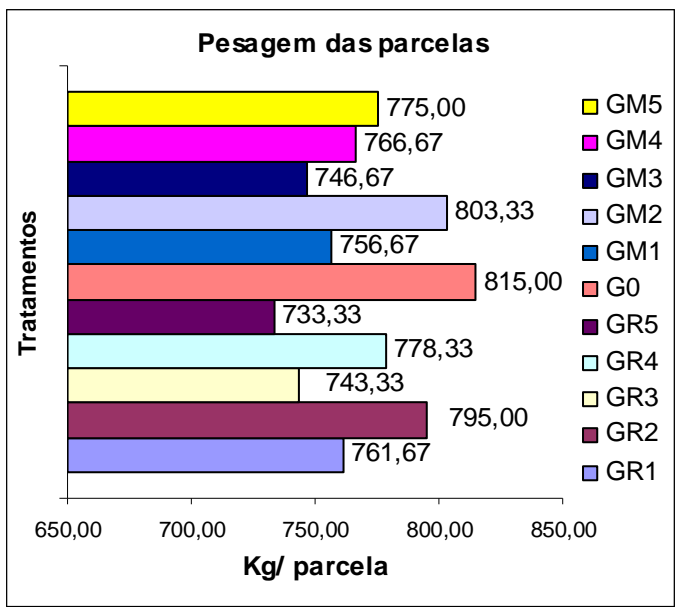

Figura 4. Valores da pesagem das parcelas referente a cada tratamento.

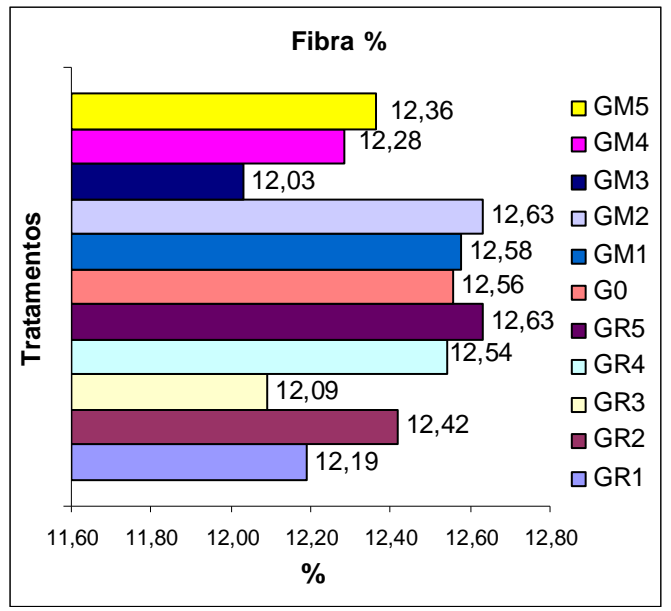

Figura 5. Teores de fibra na cana produzida referente a cada tratamento. 
Os resultados demonstram que nem sempre o acréscimo quantitativo da produção agrícola corresponde ao acréscimo proporcional de produção industrial, necessitando da comprovação de qualidade da matéria prima que está sendo entregue a indústria.

Como exemplo tem-se os resultados dos tratamentos G0, GM2 e GR2. Neles foram obtidos os maiores valores em peso de cana de açúcar, porém, obtendo também teores entre os mais altos de fibra.

Altos teores de fibra podem ter efeito negativo na produção sucroalcooleira, pois a fibra retém parte do caldo na etapa de prensagem da cana e extração do caldo, que futuramente resultaria em açúcar e álcool.

Os resultados apresentados nos gráficos das figuras 6 e 7 quantificam o ATR, açúcares redutores, teoricamente recuperável pelas usinas e atual moeda de negociação de cana segundo o modelo proposto pelo CONSECANA, e a TPH que quantifica basicamente a tonelada de sacarose contida no colmo, no caldo, no xarope, na massa cozida, etc. da cana colhida por hectare.

Com relação à ATR destacaram-se os tratamentos GM1, GR4, GM5 e GM2 com as maiores quantidades de ATR na cana que consequentemente alcançariam melhor remuneração por tonelada de cana produzida.

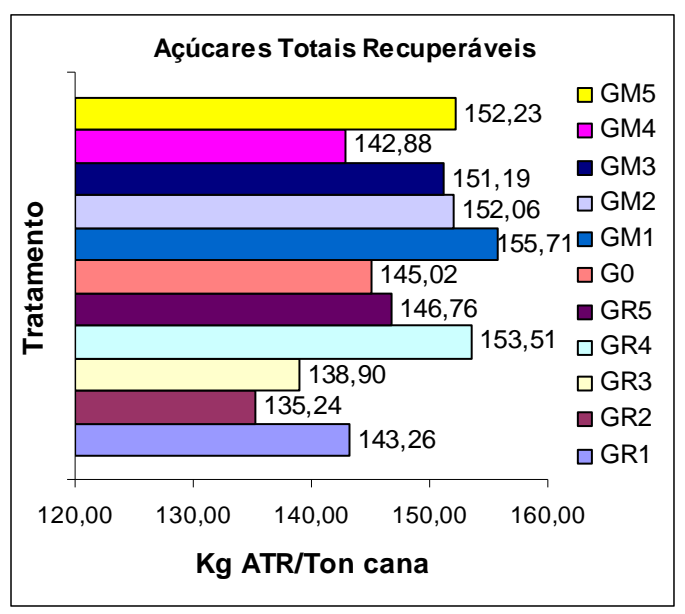

Figura 6. Valores de ATR na cana-deaçúcar

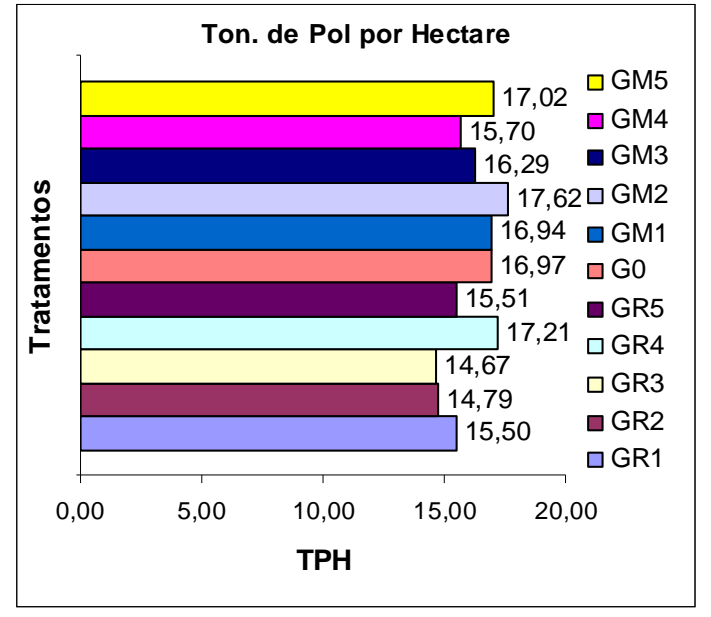

Figura 7. Valores de TPH na cana-deacúcar em seus resnectivos tratamentos.

No caso da TPH os resultados obtidos não apresentaram diferenças expressivas entre as fontes e doses utilizadas. Podendo ser destacados os tratamentos GM2 e GR4 demonstrando, para ambos, que além de ter uma boa recuperação teórica de açúcares produzidos (ATR), boa parte desse açúcar é composta por sacarose, quantificada no gráfico pela TPH. 
A sacarose é a principal molécula de açúcar utilizada na fabricação do açúcar cristal por ter características químicas como tamanho e estabilidade de sua molécula, que conferem um melhor rendimento industrial da matéria prima.

\section{CONCLUSÃO}

Pela ausência de diferença estatística entre os tratamentos constata-se a similaridade das fontes de gesso e a possibilidade de utilização do Gesso Reciclado (GR) como corretivo, assim como o Gesso Mineral (GM).

Além de avaliações quantitativas comprova-se a necessidade de avaliações qualitativas da cana-de-açúcar, para real avaliação de rendimento agro-industrial da atividade sucroalcooleira.

A dose 2 do Gesso Mineral (GM2) demonstrou a melhor viabilidade de utilização, pois com uma das menores doses de gesso foram obtidos rendimentos sempre entre os maiores, comparativamente aos outros tratamentos.

\section{REFERÊNCIAS}

ABNT - ASSOCIAÇÃO BRASILEIRA DE NORMAS E TÉCNICAS. NBR 6946/87 Materiais refratários: determinação granulométrica por peneiramento de matérias primas refratárias e refratários não conformados. Rio de Janeiro, 1991.

CAIRES, E.F. et al. Produção de milho, trigo e soja em função das alterações das características químicas do solo pela aplicação de calcário e gesso na superfície em sistema de plantio direto. Revista Brasileira de Ciência do Solo. Viçosa, Minas Gerais, v.23, p.315327, 1999.

Cana-de-açúcar. Disponível em: http://br.geocities.com/atine50/cana/cana.htm . Acesso em: 12 abr. 2008.

CAVALCANTI, F.J.A. (coord). Boletim de recomendações de corretivos e fertilizantes para o estado de Pernambuco. Recife, Pernambuco, $2^{a}$ aproximação. $2^{a}$.ed. rev., IPA, 1998. p.198.

QUAGGIO, J. A. Acidez e calagem em solos tropicais. Campinas: Instituto Agronômico, 2000. p.111.

LANDELL., M.G.A. et al. Oxisol subsurface chemical attributes related sugarcane productivity. Scientia agrícola, v 60, p 741-745, 2003.

RAIJ, B. Van et al. Gesso na produção de cultivares de milho com tolerância diferencial a alumínio em três níveis de calagem. Revista Brasileira de Ciência do Solo. Viçosa, v.22, p.101-108, 1998. 